\title{
Die Adressatinnen und Adressaten am Beispiel von Korinth
}

\begin{abstract}
Nach den Beschreibungen der Ausgangskultur und des Paulus als Vermittler eines kulturellen Artefakts kommt im Folgenden die Adressatenschaft als Zielkultur des Vermittlungsgeschehens in den Fokus. Zunächst erfolgt ein allgemeiner Überblick über die paulinische Adressatenschaft, wie sie sich aus den paulinischen Briefen rekonstruieren lässt. Sodann erfährt die Adressatenschaft in Korinth eine eingehende Untersuchung. Diese ist auch die Grundlage für die detaillierte Auslegung von 1 Kor 9,19-23 und 24-27, wo Paulus sich zunächst selbst als interkulturellen Vermittler darstellt und dieses Vermitteln in unmittelbarer Folge mit einem anschaulichen Beispiel aus der realen Lebenswelt der Adressatinnen und Adressaten umsetzt.
\end{abstract}

Wer sind Paulus' Adressatinnen und Adressaten? Textimmanente Hinweise deuten hinsichtlich der intendierten Adressatenschaft unmissverständlich zu den Menschen aus den Völkern. Dies zeigt sich schon in 1 Thess 1,9, wo Paulus bereits auf den Erfolg seiner Mission unter den Völkern zurückblickt. Auch in Gal 1,16 betont er innerhalb des Berichts über seine Berufung, Gott habe in ihm seinen Sohn offenbart, damit er ihn unter den Völkern verkündige:

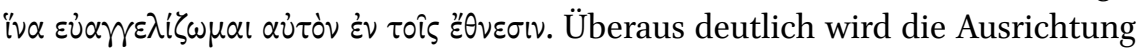
der paulinischen Mission unter den Völkern sodann im Bericht über die $\mathrm{Zu}$ sammenkunft des Paulus mit den Aposteln in Jerusalem, wo er diesen vorlegt,

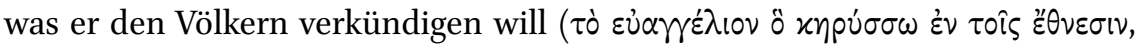
Gal 2,2). In Jerusalem wird Paulus' Eigenständigkeit als Apostel für die Völker bestätigt (Gal 2,7-9). ${ }^{1}$ Während Petrus, Jakobus, Kephas und Johannes ihre Mission auf die Beschnittenen ausrichten, gehen Paulus und Barnabas zu den Nationen ( den Unbeschnittenen (Gal 1,16; 2,7-9; Röm 11,13) - was als Synonym zu verstehen ist - einerseits und andererseits die Tatsache, dass Paulus an Heiden schreibt und diese auch direkt anspricht (Röm 1,5-6.13; 11,13; 15,16), deuten auf

1 Vgl. Apg 15,1-33. 
eine intendierte Adressatenschaft von primär nicht jüdischer Provenienz hin. So wendet sich Paulus im Präskript seines Briefes nach Rom denn prominent als Völkerapostel an alle Völker, die Berufene Jesu Christi sind: $\delta \imath^{\prime}$ o $\tilde{\varepsilon} \lambda \lambda \dot{\alpha} \beta 0 \mu \varepsilon v$

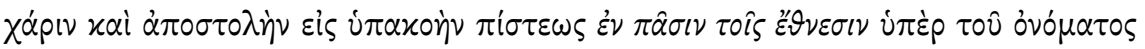

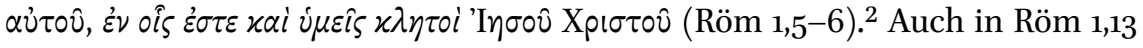
spricht er die Adressatinnen und Adressaten als Menschen aus den Völkern an, die er gerne bereits besucht hätte, und hebt in direkter Anrede an seine Adressatinnen und Adressaten ihre Herkunft und damit implizit seine Identi-

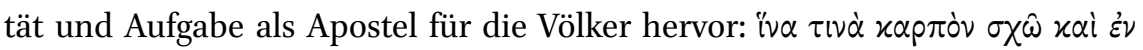

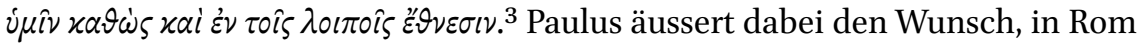
ebenso wie unter den übrigen Völkern einige Frucht zu haben. In Röm 11,11-24

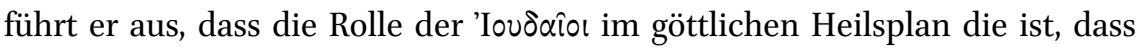
durch ihr Straucheln das Heil auch zu den Völkern komme. Dabei richtet Paulus die Argumentation in direkter Rede an sie. Und schliesslich scheint sich die Aufforderung, einander anzunehmen, in Röm 15,7 aufgrund der Argumentation in den folgenden beiden Versen insbesondere an Christusgläubige aus den Völkern zu richten. Auch in Röm 15,14-21 findet sich das Motiv der Verkündigung unter den Völkern noch einmal sehr ausführlich. Auf den Punkt gebracht und zusammenfassend kann mit den Worten von Lloyd Gaston festgehalten werden: „Paul writes to Gentile Christians, dealing with Gentile Christian problems, foremost among which was the right of Gentiles qua Gentiles, without adopting the Torah of Israel, to full citizenship in the people of God.“4

Im Gegensatz zu den zahlreichen textimmanenten Hinweisen sind Quellen über die realen Rezipienten sehr spärlich. Grundsätzlich herrscht in der Forschung Konsens, dass viele Menschen aus den Völkern, die sich von Paulus ansprechen lassen, nicht ausschliesslich in paganen Praktiken und Traditionen zu Hause sind, sondern als Proselyten oder Gottesfürchtige bereits in einer näheren Beziehung zum Judentum stehen. ${ }^{5}$ Wiederum textimmanent ist als

2 Hierzu bemerktJ. D. G. Dunn, dies sei:„probably one of the clearest indications that the Roman congregations were largely gentile, and probably mostly gentile [...].“ James D. G. Dunn, Romans, 2 Bde., WBC 38A-B (Dallas (TX) 1988), 19, Hervorhebung im griechischen Text EK.

3 Kursive Hervorhebung EK.

4 Lloyd Gaston, Paul and the Torah (Vancouver 1987), 23. Vgl. auch Stanley Kent Stowers, $A$ Rereading of Romans:Justice, Jews, and Gentiles (New Haven, London 1994), 29-33; Fredriksen, Paul, 2.

5 Vgl. dazu jüngst ebd., 54-57 und 65-69. Zum Thema der Proselyten/Gottesfürchtigen vgl. auch Bernard J. Bamberger, Proselytism in the Talmudic Period (New York 1968); Folker Siegert, „Gottesfürchtige und Sympathisanten“, in: JSJ 4, Nr. 2 (1973), 109-164; Schürer, The History of the Jewish People in the Age of Jesus Christ, 150-176; Louis H. Feldman, Jew and Gentile in the 
Beispiel Röm 7,1 anzuführen, wo sich Paulus an Menschen wendet, die über

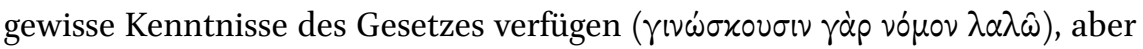

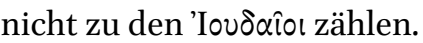

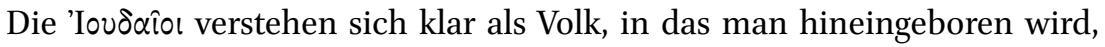
sie orientieren sich demnach an einer biologischen Abstammung. Die

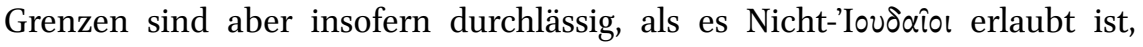
auch ohne Konversion nach den jüdischen Sitten und Gebräuchen zu leben

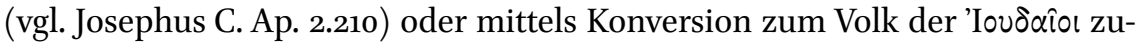
gehörig zu werden. Dies bedeutet einen radikalen Schritt. ${ }^{6}$ Für diese nicht

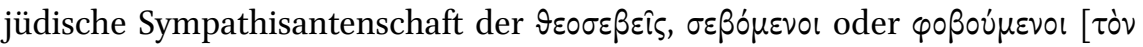
$\theta$ còv] (lat. metuentes) gibt es innerbiblisch zahlreiche Hinweise. ${ }^{7}$ Insbesondere

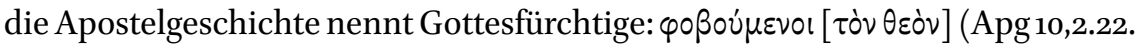

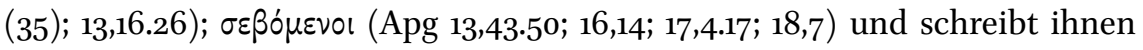
im Zusammenhang der Ausbreitung des Glaubens an Jesus als den Christus eine wichtige Brückenfunktion zwischen 'Iovסðîor und Menschen aus den

Ancient World: Attitudes and Interactions from Alexander to Justinian (Princeton (NJ) 1993); Martin Goodman, Mission and Conversion: Proselytizing in the Religious History of the Roman Empire (Oxford 1994) (speziell für das 1.Jh. n. Chr. siehe S. 60-90); Bernd Wander, Gottesfürchtige und Sympathisanten: Studien zum heidnischen Umfeld von Diasporasynagogen, WUNT 104 (Tübingen 1998); Daniel R. Schwartz, „Doing Like Jews or Becoming a Jew? Josephus on Women Converts to Judaism“, in: Frey, Schwarz und Gripentrog, Jewish Identity in the Greco-Roman World, 93-109.

6 Vgl. dazu Fredriksen: „Such a decision, given ancient divinity's ethnic embededness, scarcely made sense: it went against a commonsense construal of ,blood; of peoplehood (thus of social identity), and of ancestral custom (thus of piety). [...] Forging an exclusive commitment to a foreign god, however - an act unique to Judaism in the pre-Christian period was tantamount to changing ethnicity. A pagan's ,becoming' a Jew in effect altered his past, reconfigured his ancestry, and cut his ties with his own patrimony, both human and divine." Fredriksen, Paul, 54-55, Hervorhebung im Original.

7 Zur Terminologie der griechischen Wortstämme siehe Fitzmyer, A Wandering Aramean,

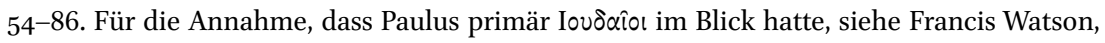
Paul, Judaism, and the Gentiles: Beyond the New Perspective, rev. and expanded ed. (Grand Rapids (MI) 2007), 167-191, ausdrücklich 188: „Romans includes Jewish Christians among its addressees." Watson relativiert damit seine frühere These, dass der Römerbrief sich vornehmlich an "Jewish Christians" richte; Francis Watson, Paul, Judaism and the Gentiles: A Sociological Approach, MSSNTS 56 (Cambridge 1986), 103. Vgl. auch Steve Mason, „Paul, Classical Anti-Judaism, and Romans", in: David J. Hawkin und Tom Robinson (Hg.), SelfDefinition and Self-Discovery in Early Christianity: A Study in Changing Horizons, SBEC 26 (Lewiston 1990), 181-223. 
Völkern zu. ${ }^{8}$ Dass es Gottesfürchtige gegeben hat, lässt sich auch durch Inschriften belegen. ${ }^{9}$

Es handelt sich bei den Gottesfürchtigen um eine wenig einheitliche Gruppe von Menschen, die mit dem Judentum zwar sympathisieren, die jüdischen Riten mehr oder weniger einhalten, aber keine formale Konversion vollzogen haben, aufgrund derer sie Mitglieder einer Gruppe geworden wären. ${ }^{10}$ Die Begriffe dieser am Judentum interessierten und dem Judentum zugewandten Menschengruppen lassen sich nicht scharf voneinander abgrenzen oder einer Klasse zuordnen. Siegert unterscheidet Gottesfürchtige von Sympathisanten. Gottesfürchtige definiert er als „ernsthaft an der jüdischen Religion Interessierte“, während er Sympathisanten als „bloße Nachahmer irgendwelcher jüdischer Bräuche oder politisch den Juden wohlgesonnene Personen" ansieht.. ${ }^{11}$ Unterschiede zwischen Gottesfürchtigen und Proselyten und wie die eine Kategorie in die andere übergehen kann, werden deutlich in Juvenals 14. Satire, 96-104. Juvenal beschreibt hier einen Vater, der den Sabbat hält und kein Schweinefleisch isst. Seine Söhne gehen indes weiter: Sie lassen sich beschneiden und studieren die jüdischen Gebote. Ein Überblick über ver-

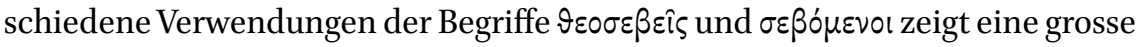
Bandbreite von Hinwendungen zum Judentum und dass es nicht möglich ist, eine einheitliche Gruppe von Gottesfürchtigen zu definieren. Was sich aus den Quellen ergibt, ist ein heterogenes Bild von unterschiedlichen Graden der $\mathrm{Zu}$ wendung zum Judentum und keine Klasse mit klar geregelten Anforderungen und definierter Zugehörigkeit zu einer Synagoge. ${ }^{12}$ Dies spiegelt sich auch in der eben erwähnten Erzählung Juvenals. Zahlreiche Forschende vermuten,

8 Vgl. Judith M. Lieu, „The Race of the God-fearers“, in: Journal of Theological Studies 46, Nr. 2 (1995), 483-501, hier: 483.

9 Vgl. Wander, Gottesfürchtige und Sympathisanten, 87-137, insbesondere die Inschrift von Aphrodisias, 235-239. Zur Aphrodisiasinschrift im Besonderen vgl. auch Joyce Maire Reynolds und Robert Tannenbaum,Jews and God-fearers at Aphrodisias: Greek Inscriptions with Commentary, PCPhS.S 12 (Cambridge 1987); Louis H. Feldman, „Proselytes and ,Sympathizers' in the Light of the New Inscriptions from Aphrodisias“, in: REJ 148, Nr. 3 (1989), 265-305.

10 Vgl. bspw. Karl G. Kuhn und Hartmut Stegemann, „Art. Proselyten“, in: Paulys RealEncyclopädie der classischen Alterthumswissenschaft. Supplement, Bd. 9 (Stuttgart 1962), $1248-1283$.

11 Siegert, „Gottesfürchtige und Sympathisanten“, 110.

12 Vgl. John Joseph Collins, Seers, Sybils and Sages in Hellenistic-Roman Judaism, JSJ.S 54 (Leiden 1997), 228-233. 
dass mehr Frauen als Männer als Gottesfürchtige Anschluss an eine Synagoge suchen. ${ }^{13}$ Für sie ist naturgemäss die Frage der Beschneidung obsolet.

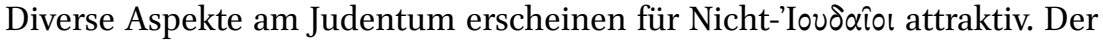
jüdische Monotheismus wirkte ebenso anziehend wie die jüdische Ethik. Insbesondere Menschen aus den unteren Schichten können von den für die Antike überdurchschnittlich ausgebauten Sozialsystemen jüdischer Gruppen profitieren. Umgekehrt kann es für Höhergestellte sinnvoller sein, im Kreis der Sympathisanten zu bleiben und nicht Proselyten zu werden, um gleichwohl den Ansprüchen der nicht jüdischen Gesellschaft gerecht werden zu können. ${ }^{14}$ In manchen Punkten passen sie sich an die jüdischen Gepflogenheiten an, durchaus in unterschiedlichem Masse, und partizipieren am synagogalen Leben, nach Möglichkeit insbesondere an den Versammlungen am Sabbat. Gelegentlich leisten sie finanzielle Zuwendungen an die Gruppen oder nutzen im Konfliktfall ihren Einfluss bei den römischen Behörden.15

Dass Paulus' Adressaten auch oder sogar primär in Kreisen von Proselyten und Gottesfürchtigen zu suchen sind, legt sich nahe durch die Tatsache, dass Paulus für seine theologischen Argumentationen eine gewisse Vertrautheit mit dem Alten Testament voraussetzt. ${ }^{16}$ Explizit nennt er diese Voraussetzung

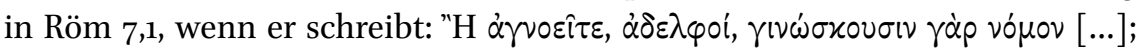
auch hinsichtlich der Adressatenschaft in Korinth lässt sich solch eine explizite Voraussetzung erkennen, was im Laufe des nächsten Abschnitts zu diskutieren ist.

\section{$5 \cdot 2$ \\ Die Adressatinnen und Adressaten in Korinth}

Bevor auf Paulus' textimmanente Hinweise hinsichtlich seiner Adressatenschaft in Korinth eingegangen werden kann, sind einige Ausführungen zur Stadt Korinth zu seiner Zeit angezeigt: ein Abriss über den historischen Kontext

13 Vgl. Kuhn und Stegemann, „Art. Proselyten“, in: Paulys Real-Encyclopädie der classischen

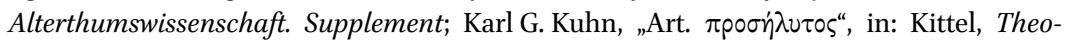
logisches Wörterbuch zum Neuen Testament, Bd. 6, 1959, 727-745.

Harnack schreibt allgemein über eine grössere Aufgeschlossenheit von Frauen gegenüber der Synagoge: Adolf von Harnack, Die Mission und Ausbreitung des Christentums in den ersten drei Jahrhunderten, 4., verb. und vermehrte Aufl. (Leipzig 1924), 589-611.

14 Als Beispiel sei an dieser Stelle der Hauptmann Kornelius genannt (Apg 10,2).

15 Als Beispiel für die finanzielle Unterstützung vgl. den Hauptmann, der nach Aussage der jüdischen Ältesten gegenüber Jesus eine Synagoge hat bauen lassen $(\operatorname{Lk} 7,5)$.

16 Vgl. Johnson Hodge, If Sons, then Heirs, 63. Zur These von Gottesfürchtigen als impliziten Adressaten bei Paulus vgl. auch Stowers, A Rereading of Romans, 21-22, 30-33, 71-72 und 277-278. 
der Zielkultur, in den zwei wesentliche Briefe der paulinischen Korrespondenz hineingerichtet sind und demgegenüber Paulus sich als Vermittler profiliert.

\subsubsection{Zur Stadt Korinth}

Das alte Korinth wird 146 v. Chr. durch den römischen Konsul L. Mummius und sein Heer zerstört und erst gut hundert Jahre später, 44 v. Chr., von Caesar als römische Colonia Laus Julia Corinthensis neu gegründet. Die Neugründung erfolgt nicht zuletzt aus wirtschaftlichen Gründen. ${ }^{17}$ Mit seiner Lage an zwei Meeren - bimaris in der Formulierung des Horaz (Hor. carm. 1.7.2) oder des Ovid (Ov. met. 5.407) - hat Korinth auch zwei Häfen zur Verfügung: Lechaion im Westen des Golfs von Korinth und Kenchreä südöstlich am Saronischen Golf. An der schmalsten Stelle sind die beiden Golfe nur durch eine 6,3 $\mathrm{km}$ breite Landenge, Isthmos genannt, voneinander entfernt und ausserdem durch eine Strassenanlage (Diolkos) verbunden. ${ }^{18}$ So entwickelt sich Korinth schnell zu einem wichtigen Handelsumschlagplatz. Insbesondere die Seeverbindung mit Alexandria ist von grosser Bedeutung. Korinth blüht nach der Zerstörung schnell wieder auf und wird von Strabo als "die reiche" bezeichnet

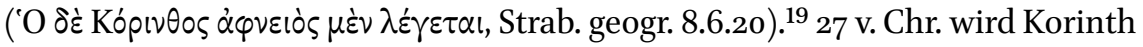
zum Sitz des Statthalters der Provinz Achaia und 44. n. Chr. zur senatorischen Provinz. Mit einer Gesamtbevölkerung von geschätzt 100.00o Einwohnern ist Korinth zur Zeit des Paulus eine grosse Stadt und hat in seiner Blüte mehr Einwohner zu verzeichnen als Athen. ${ }^{20}$ Zur Zeit von Paulus' Aufenthalt in Korinth ist auch das von der Stadtmauer umfasste Gebiet zweieinhalbmal so gross wie Athen. ${ }^{21}$

Nicht nur von der Grösse, sondern auch vom kulturellen Leben her ist Korinth eine bedeutende Stadt. Auf dem Isthmos am Eingang zur Peloponnes werden im Poseidonheiligtum alle zwei Jahre wichtige Wettkämpfe ausgetragen: die Isthmischen Spiele. Zwischen 7 v. Chr. und 3. n. Chr. kehren die Spiele wieder zurück zum Isthmischen Heiligtum, nachdem sie aufgrund der

17 Dass die Zerstörung komplett war, könnte auch römische Propaganda sein; vgl. Cic. Tusc. 3.53, wo zwar von den zerstörten Stadtmauern berichtet wird, aber auch von einer verbliebenen Bevölkerung.

18 Strabo beschreibt, wie Schiffe über einen gepflasterten Ziehweg über den Diolkos gezogen wurden, um die 40 Stadien bis zum anderen Meeresarm zu überwinden: Strab. geogr. 8.2.1; 8.6.4; 8.6.22. Dies ersparte eine Umsegelung der Peloponnes. Zum Diolkos siehe auch Jerome Murphy-O'Connor, „The Corinth That Saint Paul Saw“, in: BA 47, Nr. 3 (1984), 147-159, hier: 149 .

19 Selbst wenn Strabo hier aus Hom. Il. 2.570 zitiert, will er die Situation Korinths zur Zeit seines eigenen Lebens beschreiben.

20 Vgl. Klauck, 1. Korintherbrief, 6. Davon sind geschätzt ein Drittel Sklaven.

21 Vgl. John E. Stambaugh, Das soziale Umfeld des Neuen Testaments, GNT 9 (Göttingen 1992), 153 . 
Zerstörung Korinths gut ein Jahrhundert lang in der benachbarten Stadt ausgerichtet worden sind. ${ }^{22}$ Zusammen mit den Spielen von Olympia, Delphi und Nemea bilden die Isthmischen Spiele die vier klassischen Panhellenischen Agone (Wettkämpfe). Nebst den Isthmischen Spielen und dem Handel scheint die Prostitution eine zentrale Einkommensquelle gewesen zu sein (vgl. Strab. geogr. 8.6.20). ${ }^{23}$ Von Athen aus wird jedenfalls der Ruf der Lasterhaftigkeit Korinths fleissig gepflegt. ${ }^{24}$ Hingegen spricht Ailios Aristides in seinen Orationes durchaus mit Anerkennung von der "Stadt der Aphrodite“, wobei er nicht auf sexuelle Perversion hinweist, sondern die Attribute der Schönheit und Liebe hervorhebt (Aristeid. Or. 46.25). Plausibel erscheint die Einschätzung von Murphy-O'Connor, der zufolge die Lasterhaftigkeit Korinths mehr in athenischer Propaganda als in der Realität zu finden sei und dass Korinth sich diesbezüglich nicht von anderen mediterranen Hafenstädten unterscheide. ${ }^{25}$

Grösse und Bedeutung von Korinth dürften also gewichtige Gründe sein, weshalb Paulus hier Fuss fasst, um sein Evangelium zu verbreiten. Korinth ist zur Zeit des Paulus eine römische Stadt, was die Architektur und die Mehrzahl der Inschriften bezeugen. ${ }^{26}$ Kaiser Augustus lässt nebst dem Theater, der nordwestlichen Stoa mit Läden auf der Agora und weiteren Läden an der Westseite der Lechaeon-Strasse auch eine Basilika und einen monumentalen Kalksteinbogen am Eingang zur Agora bauen. Auch unter den Kaisern Tiberius, Gaius und Claudius wird im grossen Stil gebaut. Die zweiteilige Agora, die an Grösse ihresgleichen sucht, zeugt davon. ${ }^{27}$ Oberhalb des anstelle der griechischen Platzanlage errichteten Forums ist ein grosser Kultbezirk entstanden, der vermutlich der kapitolinischen Trias Minerva, Jupiter und Juno geweiht ist und in dessen Nähe sich auch das macellum befindet, wo Fleisch und Fisch verkauft

22 Vgl. Martin Brändl, Der Agon bei Paulus: Herkunft und Profil paulinischer Agonmetaphorik, WUNT II 222 (Tübingen 2006), 225, mit Verweis auf eine lateinische Inschrift: „agonothete iSTHMION ET CAESAR eon qui isthmIA AD ISTHMUM EGIT primus sub curaM COL LAUD IUL. COR."; vgl. die Inschrift bei John H. Kent (Hg.), The Inscriptions 1926-1950, Corinth, Vol. 8, Pt. 3 (Cambridge (MA) 1966), 70, Nr. 153, Z. 6-8.

23 Zu beachten ist allerdings, dass Strabon (63 v. Chr.-19 n. Chr.) sich auf die "goldene Zeit", d. h. die griechische Zeit, bezieht und dass die von ihm genannte Zahl von über 1.000 Prostituierten für das römische Korinth nicht ohne Weiteres angenommen werden kann; vgl. Hans Conzelmann, Der erste Brief an die Korinther, 11. Aufl., KEK, Abt. 5, Ed. 11 (Göttingen 1969), 25-26.

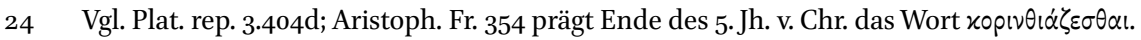

25 Vgl. Murphy-O'Connor, „The Corinth That Saint Paul Saw“, 152; Jerome Murphy-O'Connor, St. Paul's Corinth: Texts and Archaeology, 3., rev. and expanded ed., GNS 6 (2002), 55-57.

26 Vgl. David W. J. Gill, „Corinth: A Roman Colony in Achaea“, in: $B Z$ 37, Nr. 2 (1993), 259-264; Donald Engels, Roman Corinth: An Alternative Model for the Classical City (Chicago 1990).

Vgl. Meeks, Urchristentum und Stadtkultur, 104. 
werden. ${ }^{28}$ Bruce Winter hält fest: „Corinth, therefore, was not a Greek city with a Roman facade. It was conceived of, and deliberately laid out, as a thoroughly Roman colony. ${ }^{“ 29}$ Nebst der römischen Architektur spricht der überdurchschnittlich grosse Anteil italischer Töpfererzeugnisse für ein römisches Gepräge. Auch numismatische Zeugnisse unterstreichen die romanitas von Korinth: Alle Münzen, die von lokalen Magistraten in der korinthischen Münzstätte bis zu deren Schliessung im Jahr $67 \mathrm{n}$. Chr. geprägt werden, sind lateinischsprachig. ${ }^{30}$ Zudem sind die aus dem 1.Jh. überlieferten Inschriften zum grossen Teil in lateinischer Sprache abgefasst. Bruce Winter kommt in seiner Untersuchung zur Lage in Korinth nach Paulus' Abreise zu dem Schluss, dass das kulturelle Milieu von romanitas geprägt sei:

In conclusion, whether rich or poor, bond or free, the cultural milieu which impacted life in the city of Corinth was Romanitas. This does not mean that there were no ethnic minorities, but it does mean that the dominant and transforming cultural influence was Roman. ${ }^{31}$

Dieser Befund, insbesondere hinsichtlich der Sprache, sollte aber nicht überbewertet werden, denn:

Die Tatsache, daß sich das Verhältnis von lateinischen zu griechischen Inschriften unter der Herrschaft Hadrians plötzlich umkehrt und die griechischen danach immer mehr dominieren, legt die Vermutung nahe, daß die Sprachverwendung im öffentlichen Bereich möglicherweise nicht den alltäglichen Sprachgebrauch der Bevölkerung repräsentierte. Ja, einige der früheren lateinischen Inschriften wurden von ehemaligen Freigelassenen bestellt, die verräterisch griechisch klingende Namen wie Babbius, Erastus und Cleogenes haben. ${ }^{32}$

So findet sich in einem Zeugnis von Valerius Maximus, der zur Zeit der Regierung von Tiberius (14-37 n. Chr.) schreibt, der Hinweis, dass die Magistraten gegenüber Griechen ausschliesslich auf Lateinisch zu antworten und nur mittels eines Übersetzers zu konversieren hätten (Val. Max. 2.2.2.). Dies deutet darauf hin, dass es zu dieser Zeit gerade nicht selbstverständlich ist, Lateinisch zu sprechen. Zudem ist die grosse Mehrheit an Ostraka auf Griechisch

28 Vgl. Dietrich-Alex Koch, „Die Städte des Paulus“, in: Oda Wischmeyer (Hg.), Paulus: Leben, Umwelt, Werk, Briefe (Tübingen 2012), 142-159, hier: 150-151.

29 Bruce W. Winter, After Paul Left Corinth: The Influence of Secular Ethics and Social Change (Grand Rapids (MI) 2001), 11.

30 Ausführlich hierzu Michel Amandry, Le monnayage des duovirs corinthiens, BCH.S 15 (Athènes, Paris 1988).

31 Winter, After Paul Left Corinth, 22.

32 Meeks, Urchristentum und Stadtkultur, 104-105. 
verfasst. ${ }^{33}$ Offiziell ist Korinth zwar lateinischsprachig, aber das Griechische ist unumstritten die Handels- und auch in weiten Teilen der Bevölkerung die primäre Umgangssprache. ${ }^{34}$

Literarische Texte aus der Mitte des 2.Jh., die von Korinth handeln insbesondere von Pausanias und Strabon, aber auch Apuleius und Ailios Aristides -, stellen die Stadt in Übereinstimmung mit archäologischen Funden als einen Ort dar, der von geistlicher Beweglichkeit geprägt ist. ${ }^{35}$ Neben den klassischen griechisch-römischen Göttern, die Teil des offiziellen Kultes sind, werden auch Mysterien gepflegt, und philosophische Schulen verbreiten ihre Lehren. Die Tempel und Statuen im Zentrum der Stadt sind Apollon, Aphrodite, Athene, Poseidon, Tyche und Zeus geweiht. Am Stadtrand stehen welche für Asklepius, Demeter und Kore. Auch der Isis Pelagia respektive der ägyptischen Isis sind Heiligtümer gewidmet, und für Serapis finden sich ebenfalls zwei Tempel. ${ }^{36}$ Die verschiedenen Tempel, Heiligtümer und Mysterien lassen auf ein reges Vereinsleben schliessen.

Straboberichtet,dass CaesareinegrosseAnzahlFreigelasseneralsKolonisten nach Korinth geschickt habe (Strab. geogr. 8.6.23). Durch Schenkungen an die Stadt haben sie Ämter sowie öffentliche Ehrungen erhalten, was ihnen die Möglichkeit eröffnet hat, Teil der sich in Korinth erst neu bildenden Aristokratie zu werden. ${ }^{37}$ Diesen Freigelassenen sollen sich zahlreiche „Griechen und Ausländer" angeschlossen haben, darunter auch viele Orientalen und insbesondere Juden. ${ }^{38}$ Die Zeugnisse, die eine Einschätzung der Grösse einer jüdischen Gruppe in Korinth in römischer Zeit zulassen, deuten in unterschiedliche Richtungen. Einerseits erwähnt Philo (Legat. 281-282) 'Iovסðî in Korinth, wobei Korinth nebst Argos als einzige Stadt genannt ist bei sonstiger Erwähnung ganzer Länder und Provinzen. Allerdings ist dies ausser der späteren Erwähnung von Hebräern in Korinth durch Justin den Märtyrer

33 Der Hinweis hierauf findet sich bei Winter, After Paul Left Corinth, 14 (mit Verweis auf Kent, The Inscriptions 1926-1950). Kent wertet den Befund allerdings ohne weitere Begründung so, dass dieser die Romanitas gerade nicht relativiere.

34 Dies konzediert selbst Winter, After Paul Left Corinth, 25.

35 Für eine Zusammenstellung der literarischen Texte siehe Murphy-O'Connor, St. Paul's Corinth.

36 Murphy-O'Connor, „The Corinth That Saint Paul Saw“, 150 und 154.

37 Herausragendes Beispiel hierfür ist Cn. Babbius Philinus, der im Amt des Ädils grosse Stiftungen leistet und daraufhin zum pontifex und duumvir avanciert. Vgl. Allen Brown West, Latin Inscriptions: 1896-1926, Corinth 8, Pt. 2 (Cambridge (MA) 1931), 107-108.

38 Vgl. Harold N. Fowler, „Introduction“, in: Harold N. Fowler und Richard Stillwell (Hg.), Corinth: Introduction, Topography, Architecture, 2 Bde., Corinth 1.1 (Cambridge (MA) 1932), 3-17, hier: 16. Im Englischen: „Greeks and foreigners“. 
das einzige literarische Zeugnis (Iust. Mart. dial. 1.3). Die spärlichen archäologischen Zeugnisse jüdischen Lebens stammen allesamt aus späterer Zeit. ${ }^{39}$

Missionsstrategisch dürfte Korinth in mancherlei Hinsicht anderen griechischen Städten überlegen sein. Durch seine geografische Lage ist Korinth zur Zeit des Paulus eine blühende Handelsstadt. Das weitgefächerte Spektrum an Kulten und die allgemeine Toleranz sprechen für eine Offenheit innerhalb der örtlichen Zielkultur und dürften der Ausbreitung des Glaubens an Jesus als kulturelles Artefakt zuträglich sein..$^{40}$ Nicht zu unterschätzen ist die Rolle, die die Isthmischen Spiele innehaben. Der Betrieb rund um diese Spiele dürfte über die allgemeinen Möglichkeiten städtischen Lebens hinaus für Paulus ein ideales Umfeld bieten, um mit Menschen zu interagieren und missionarisch zu wirken. ${ }^{41}$ Im Folgenden werden nun textimmanente Hinweise zur Beschreibung der Adressatenschaft in Korinth diskutiert.

\subsubsection{Textimmanente Hinweise}

Im ersten Schreiben an die Christusgläubigen in Korinth wendet sich Paulus

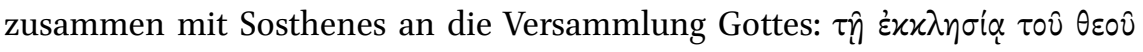
$\tau \hat{\eta}$ oủ Geheiligten. Ausserdem wendet er sich an alle, die an jedem Ort den Namen „unseres Herrn“ Jesu Christi anrufen (1 Kor 1,2). In 1 Kor 6,11 spricht Paulus die Adressatinnen und Adressaten ganz direkt an als solche, von denen manch einer ein Laster überwunden hat (vgl. 1 Kor 6,9-10) und jetzt abgewaschen

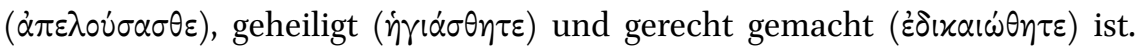
Die Quellen für die Darstellung der korinthischen Gruppe sind sowohl hinsichtlich ihrer ethnischen Zusammensetzung als auch ihrer übrigen sozialen Schichtung sehr spärlich. Die soziale Schichtung betrifft neben der ethnischen

39 Die Abbildung auf der Terracottalampe No. 1511 könnte ein siebenarmiger Leuchter sein, stammt aber aus dem 5. oder frühen 6. Jh. n. Chr. Und auch die Inschrift, die auf eine Synagoge von Hebräern hinweist und auf der Lechaeum-Strasse gefunden wurde, ebenso wie einige hebräische Grabsteine sind auf Jahrhunderte später als das 1. Jh. zu datieren; vgl. Oscar Theodore Broneer, Terracotta Lamps, Corinth 4.2 (Cambridge (MA) 1930), 121122. Zur Synagogeninschrift vgl. auch Benjamin Powell, „Greek Inscriptions from Corinth“, in: $A J A$ 7, Nr. 1 (1903), 26-71, hier: 6o-61.

40 Vgl. Helmut Merklein, Der erste Brief an die Korinther: Kapitel 1-4, 3 Bde., ÖTBK 7.1 (Gütersloh, Würzburg 1992), 28.

41 Vgl. auch Brändl, Der Agon bei Paulus, 242. Zur Stadt als Ort von paulinischer Mission im Allgemeinen vgl. Meeks, Urchristentum und Stadtkultur, 24-26; Martin Ebner, Die Stadt als Lebensraum der ersten Christen, Grundrisse zum Neuen Testament. Ergänzungsreihe 1, 1 (Göttingen 2012); von Bendemann und Markus Tiwald, „Das frühe Christentum und die Stadt - Einleitung und Grundlegung“, in: von Bendemann, Das frühe Christentum und die Stadt, 9-42. 
Zusammensetzung und dem ordo (d.h. der Zugehörigkeit zu einer Klasse) auch die Geschlechterverteilung, die persönliche Freiheit der Mitglieder, ihr Vermögen und Alter, ihre Ämter, Würden, Berufe und Bürgerschaft, weshalb man sich für deren Beschreibung auf textimmanente Hinweise stützen muss. ${ }^{42}$ Hierfür sind neben einigen allgemeinen Begriffen insbesondere die Namen zentral, da sie soziologisch verwertbare Hinweise enthalten und Rückschlüsse auf die Gruppe zulassen. Relevant sind zusätzlich zu den Angaben aus den Briefen an die Gruppe in Korinth auch Informationen aus dem Brief an die Christusgläubigen in Rom, da dieser in Korinth verfasst ist und am Ende eine Liste der Grüssenden enthält (Röm 16,21-23). Kaum zu entscheiden ist allerdings, welche der über Paulus' Mitarbeiter Timotheus in Röm 16 hinaus genannten Personen, die aus Korinth Grüsse schicken, tatsächlich aus Korinth und welche Mitreisende des Paulus sind. Auch über die soziale Schichtung und die Grösse der Gruppe finden sich Hinweise. Zum Kontext, innerhalb dessen Briefe vorgelesen werden, gibt es ebenfalls spärliche Hinweise.

\subsubsection{Lateinische und griechische Namen}

Die Menge an lateinischen Namen ist auffällig. In 1 Kor sowie in Röm unter den aus Korinth Grüssenden nennt Paulus sechs Menschen mit lateinischen

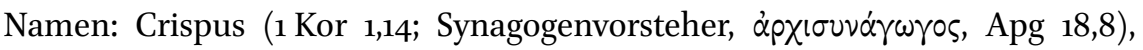
Gaius (1 Kor 1,14; vgl. Röm 16,23, wo Gaius als Gastgeber genannt ist), Fortunatus (1 Kor 16,17), Lucius (Röm 16,21), Tertius (Röm 16,22), Quartus (Röm 16,23). Hinzu kommt in der Apostelgeschichte noch Titius Justus (Apg 18,7; ein Gottesfürchtiger). Auch die Namen Priska und Aquila (1 Kor 16,19; Apg 18,2.18, wobei hier statt Priska das Diminutivum Priskilla erscheint) sind lateinisch, allerdings kommen sie bekanntlich aus Rom und ihr Aufenthalt in Korinth ist nur vorübergehend (vgl. Apg 18,2 und 18,8).

Daneben erscheinen die griechischen Namen Sosthenes (1 Kor 1,1), Stephanas (Hauseigentümer, 1 Kor 1,16; 16,15.17), Chloë („Leute der“, 1 Kor 1,11), Achaikos (1 Kor 16,17), Phoebe (aus Kenchreä, Röm 16,1), Apollos (1 Kor 1,12; 3,4.5.6.22; 4,6; 16,12), Iason und Sosipater (Stammverwandte des Paulus:

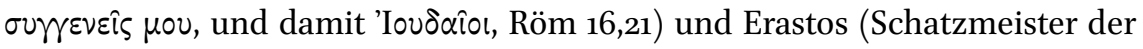

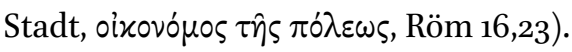

42 Für die Einstufung in einen sozialen Status verwendet Meeks folgende Kriterien: ethnische Zugehörigkeit, ordo, Bürgerschaft, persönliche Freiheit, Vermögen, Beruf, Alter, Geschlecht sowie öffentliche Ämter und Ehren; vgl. den Abschnitt „Die Messung sozialer Schichtung" in Meeks, Urchristentum und Stadtkultur, $115^{-120 .}$ 


\subsubsection{Ethnische Zusammensetzung}

Hinsichtlich der ethnischen Zusammensetzung deuten textimmanente Hinweise des Paulus darauf, dass die korinthische Gruppe primär aus Menschen aus den Völkern besteht. ${ }^{43}$ Paulus konstatiert dies, indem er die Adressatinnen

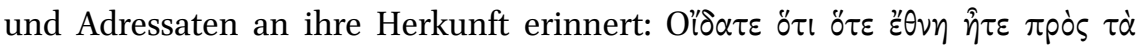

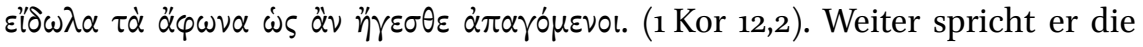
Adressatinnen und Adressaten in 1 Kor 8, insbesondere Vers 7, ebenfalls implizit als Menschen aus den Völkern an. Diese Herkunft wird auch in 1 Kor 10,14 eindeutig vorausgesetzt. Selbst die Aufforderung in 1 Kor 7,18-20, dass die als Beschnittene Berufenen bei der Beschneidung bleiben sollen, ist eher als Hinweis auf Proselyten denn auf gebürtige 'Iovס $\alpha i ̂$ o zu lesen. ${ }^{44}$ Auch 1 Kor 1,1825 und 12,13 weisen auf die Vorstellung einer gewissen Durchmischung hin.

Aus den Erwähnungen des Petrus (1 Kor 1,12; 3,22; 9,5 und 15,5) geht hervor, dass Paulus in Korinth bekannt ist. Auch die Missstände, die Paulus im 1 Kor anspricht, sind am ehesten verständlich, wenn man von einer heidnischen Vergangenheit der Adressatenschaft ausgeht. Zu nennen sind Unzucht, Stolz, Hetärenverkehr, Prozessieren vor heidnischen Gerichten, Ungerechtigkeit, Götzendienst, Ehebruch, Knabenschänderei, Diebstahl, Trunkenheit, Umgang und Mahlgemeinschaft mit Unzüchtigen, Habsüchtigen, Götzendienern, Lästerern, Trunkenbolden oder Räubern (1 Kor $5^{-6}$ ).

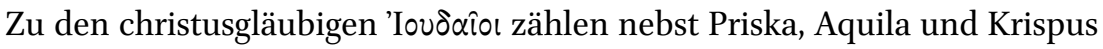
vielleicht auch Sosthenes und sicher Apollos. ${ }^{45}$ Mit Priska und Aquila gehören vgl. Apg 18,2-3 - zur Gruppe in Korinth von Beginn an auch Christusgläubige jüdischer Herkunft, aber ein Anschluss an eine Synagoge und eine Mission innerhalb derselben folgen daraus nicht unbedingt. ${ }^{46}$ Allerdings lassen die

43 Dass die Gruppe in Korinth sich überwiegend aus Menschen aus den Völkern zusammensetzt, wird in der Forschung verbreitet angenommen; vgl. bspw. Gerhard Sellin, „Hauptprobleme des Ersten Korintherbriefes“, in: Wolfgang Haase (Hg.), Principat 25, 2: Vorkonstantinisches Christentum: Leben und Umwelt Jesu; Neues Testament (Berlin 1984), 2940-3044 und 2996-2997; Udo Schnelle, Einleitung in das Neue Testament, 9., durchgeseh. Aufl. (Göttingen 2017), 79; Hans-Martin Schenke, Einleitung in die Schriften des Neuen Testaments, Lizenzausg. (Gütersloh 1978-1979), 91; Klauck, ı. Korintherbrief, 6; Peter Richardson, „On the Absence of ,Anti-Judaism ' in 1 Corinthians“, in: Peter Richardson (Hg.), Anti-Judaism in Early Christianity (Waterloo (ON) 1986), 59-74, hier: 63.

44 Vgl. Fredriksen, Paul, 107 und 181 (Fn. 3).

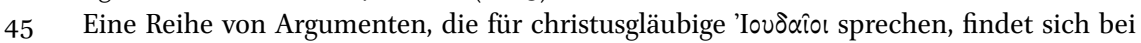
Peter Richardson, „On the Absence of ,Anti-Judaism“ in 1 Corinthians“, in: Anti-Judaism in Early Christianity, 64. Zur judenchristlichen Herkunft des Apollos siehe bspw. Schenke, Einleitung in die Schriften des Neuen Testaments, 91-92.

46 Vgl. Gerhard Sellin, „Hauptprobleme des Ersten Korintherbriefes“, in: Principat 25, 2, 2996. 
zahlreichen Schriftzitate, aber auch die Erzählung über die Wüstengeneration (1 Kor 10,1-10), darauf schliessen, dass Paulus bei seiner Adressatenschaft ein gewisses Mass an Kenntnis über die jüdischen Traditionen voraussetzt. Das "wir", das sich in 1 Kor 10,1 auf „unsere Väter" bezieht, könnte alle potenziellen Christusgläubigen in Korinth meinen und sie somit in die Geschichte des Volkes Israel mit einbinden. ${ }^{47}$ Ausserdem weist Paulus mehrfach auf Kephas hin (1 Kor 1,12; 3,22; 9,5; 15,5), der bekanntlich ausschliesslich für die Verkündigung des Evangeliums unter den 'Iovס̊aiol zuständig ist (Gal 2,7-9). Paulus scheint bei seinen Erwähnungen des Kephas vorauszusetzen, dass er der Adressatenschaft bekannt ist.

Nach der Darstellung der Apostelgeschichte (Apg 18,4) predigt Paulus bei seinem Eintreffen in Korinth, wie auch an anderen Orten, zuerst in der örtlichen Synagoge. Der Apostelgeschichte zufolge führen seine Gespräche in der Synagoge zu Auseinandersetzungen mit den Mitgliedern derselben (Apg 18,4-6) und zur Anzeige beim Statthalter Gallio (Apg 18,12-16).48 Das Bild, das die Apostelgeschichte zeichnet, steht in einer gewissen Spannung zu den paulinischen Aussagen, nach denen er seine Mission, wenn nicht ausschliesslich, so auf jeden Fall primär, an die Menschen aus den Völkern richtet (Gal 1,16; 2,7-9; Röm 1,5.13-15; 11,13-14; 15,15-21). Die gottesfürchtigen Menschen aus den Völkern, auf die seine Mission am ehesten zielen dürfte, sind allerdings

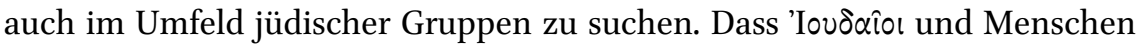
aus den Völkern, zumal Gottesfürchtige, nicht segregiert voneinander lebten, hat jüngst Paula Fredriksen hervorgehoben. ${ }^{49}$

Die christusgläubige Gruppe hat Paulus nach eigenen Angaben selbst gegründet (explizit 1 Kor 4,15; sinngemäss auch 2,1-5; 3,5-6; vgl. 2 Kor 1,19: hier nennt Paulus die Unterstützung durch Silvanus und Timotheus; vgl. Apg 18,5). Um seine bleibende Verbundenheit mit ihr auszudrücken, benutzt Paulus Begriffe verwandtschaftlicher Beziehungen. So stellt er sich im Brief an die Christusgläubigen in Korinth als Vater dar, indem er die Adressatinnen und

47 Hierfür spricht, dass dieselben ab 1 Kor 10,8 als direkt Angesprochene mit Sicherheit mitgemeint sind, sodass sie aus der Retrospektive auch in 10,1.6 mitgemeint sein könnten; vgl. Wolff, Paulus beispiels-weise, 191 (Fn. 28).

48 Vgl. dazu Meeks, Urchristentum und Stadtkultur, 58. Hingegen relativiert Wolfang Reinbold in seiner Analyse die Rolle der „altgläugigen Juden unter den Opponenten“; vgl. Wolfgang Reinbold, Propaganda und Mission im ältesten Christentum: Eine Untersuchung zu den Modalitäten der Ausbreitung der frühen Kirche, FRLANT 188 (Göttingen 200o), 209. Ein anschauliches Beispiel für die Rekrutierung eines Christusgläubigen aus den Kreisen von Gottesfürchtigen in Korinth bietet Apg 18,7 mit Titius Justus; vgl. dazu ebd. 185 .

49 Vgl. die beiden Abschnitte "Jews in Pagan Places“ und „Pagans in Jewish Places“ in Fredriksen, Paul, 38-49 und 49-6o. 
Adressaten als seine geliebten Kinder anspricht ( $\tau \varepsilon \dot{\varepsilon} \kappa \nu \alpha \mu 0 \nu \dot{\alpha} \gamma \alpha \pi \eta \tau \dot{\alpha}, 1$ Kor 4,14)

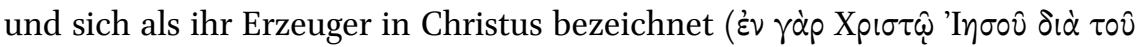

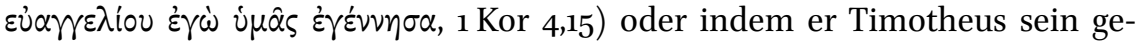

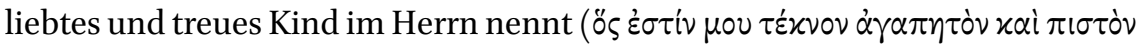

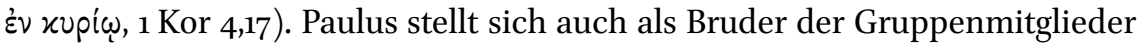
dar. Den in diesem Zusammenhang singularisierten „Bruder“ innerhalb der Gruppe will er nicht „zu Fall bringen“ und verzichtet deshalb fortan auf den Verzehr von Fleisch (1 Kor 8,13). Überdies spricht er die Adressaten als seine

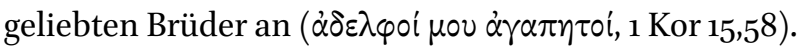

\subsubsection{Weitere Aspekte der sozialen Zusammensetzung}

Die in 1 Kor namentlich Genannten dürften mehrheitlich einer gehobenen Minderheit angehören, was aus der Erwähnung von Besitz, Dienstleistungen und Ämtern hervorgeht. ${ }^{50}$ Die Formulierung „Leute der Chloë“ (1 Kor 1,11) deutet hingegen eher auf eine Herkunft aus dem Sklavenstand hin. Ob auch Chloë selbst christusgläubig ist, muss offenbleiben. Chloë bedeutet, die Grüne und ist ein Epitheton der Korngöttin Demeter. Auch Chloë dürfte aus dem Sklavenstand kommen. ${ }^{51}$ Ähnlich lässt auch der Name Phoebe ,leuchtend, rein' (Röm 16,1) eine Herkunft aus dem Sklavenstand oder von Freigelassenen vermuten, wobei sie einen gewissen Wohlstand erlangt haben muss, um nach Rom reisen zu können und in ihrer Rolle als Dienerin der Christusgläubigen in Kenchreä den Brief des Paulus an die Christusgläubigen in Rom zu überliefern. ${ }^{2}$

Aufschluss hinsichtlich der sozialen Schichtung in Korinth gibt vornehmlich 1 Kor 1,26-28 mit der Angabe, dass in der Berufung ( $\kappa \lambda \hat{\eta} \sigma \varsigma \varsigma)$ der Angeschriebenen nicht viele Weise nach dem Fleisch, nicht viele Mächtige, nicht

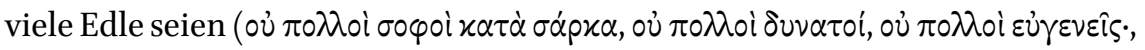
1 Kor 1,26), aber Gott habe das Törichte der Welt auserwählt ( $\tau \dot{\alpha} \mu \omega \rho \dot{\alpha} \tau o v$ xó $\sigma \mu \circ v$

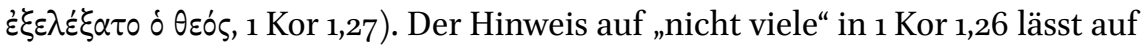
ein soziales Gefälle innerhalb der Gruppe schliessen: ein Gefälle sowohl hinsichtlich der Bildung und der gesellschaftlichen Einflussmöglichkeiten als

$5^{0}$ Vgl. Gerd Theißen, „Soziale Schichtung in der korinthischen Gemeinde: Ein Beitrag zur Soziologie des hellenistischen Urchristentums", in: Gerd Theißen (Hg.), Studien zur Soziologie des Urchristentums, 2., erw. Aufl., WUNT 19 (Tübingen 1983), 232-257.

51 Vgl. Theißen, Studien zur Soziologie des Urchristentums, 254-255; Hans-Josef Klauck, Herrenmahl und hellenistischer Kult: Eine religionsgeschichtliche Untersuchung zum ersten Korintherbrief, NTA.NF 15 (Münster 1982), 28-29. F. R. Montgomery Hitchcock hat die These aufgestellt, dass der Name Chloë als Hinweis auf den korinthischen Demeterkult zu interpretieren sei; vgl. F. R. M. Hitchcock, „WHO ARE ,THE PEOPLE OF CHLOE‘ IN i Cor. i ii?“, in:JThS 25, Nr. 98 (1924), 163-167.

Vgl. Klauck, Herrenmahl und hellenistischer Kult, 30-31. 
auch des sozialen Prestiges. Theißen rechnet damit, dass die korinthische Gruppe durch eine innere soziale Schichtung charakterisiert ist:

Einigen tonangebenden Gemeindegliedern aus der Oberschicht steht die große Zahl von Christen aus den unteren Schichten gegenüber. Diese innere Schichtung ist nicht zufällig, sondern hat strukturelle Gründe. Die soziale Zusammensetzung der korinthischen Gemeinde dürfte daher für die hellenistischen Gemeinden überhaupt charakteristisch sein. ${ }^{53}$

Gelegentliche soziale Differenzen lassen sich auch an den Ausführungen zum Fleischessen und zu den Versammlungen erkennen (1 Kor 8 bzw. 1 Kor 11,17-22). Die Tatsache, dass die Kollekte sich nach den Möglichkeiten des Einzelnen richten soll, weist auf unterschiedliche Besitzverhältnisse hin (1 Kor 16,2). Überdies gibt es deutliche Hinweise auf fundamentale Unterschiede hinsichtlich des Standes, wenn von Sklaven und von Freien die Rede ist $(1$ Kor $7,21-23 ; 12,13){ }^{54}$

\subsubsection{Zur Grösse und zum Wachstum der Gruppe in Korinth}

Auch für Schätzungen zur Grösse der Gemeinde und zu ihrem Wachstum und vor allem zur Anzahl der bei einer Versammlung Anwesenden muss man sich

53 Theißen, Studien zur Soziologie des Urchristentums, 231. Demgegenüber hat Dirk Jongkind geltend gemacht, dass es in Korinth nicht bloss eine kleine Elite und eine grosse Mehrheit von Armen gebe, sondern auch eine breitere Mittelschicht. Er argumentiert aufgrund eines Vergleichs mit der Archäologie in Pompeii, dass es auch in Korinth ein relativ breites Spektrum an Wohngelegenheiten gebe, eine Tatsache, die es verbiete, bloss von einer Elite und einer grossen Masse an ganz Armen auszugehen; vgl. Dirk Jongkind, „Corinth in the First Century AD: The Search for Another Class“, in: TynB 52, Nr. 1 (2001), 139-148.

54 Zur wirtschaftlichen Einordnung von frühen Christusgläubigen stehen unterschiedliche Einschätzungen nebeneinander. Für eine überaus grosse Mehrheit aus den ärmsten Schichten analog zur allgemeinen gesellschaftlichen Schichtung argumentieren Stegemann und Stegemann, Urchristliche Sozialgeschichte, 249-271; Justin J. Meggitt, Paul, Poverty and Survival, Studies of the New Testament and its world (Edinburgh 1998). Für einen repräsentativen Querschnitt aller Schichten ausser der höchsten und niedrigsten Stufe in den paulinischen Gemeinden plädiert bspw. Abraham J. Malherbe, Social Aspects of Early Christianity, 2nd ed., enlarged (Philadelphia 1983). Malherbe beansprucht sogar, dass diese Sicht sich zunehmend als Forschungskonsens etabliere. Vgl. dazu auch Meeks, Urchristentum und Stadtkultur, 155-157, und Theißen, Studien zur Soziologie des Urchristentums, 272-289. Eine Zwischenposition vertritt Bruce W. Longenecker, „SocioEconomic Profiling of the First Urban Christians", in: Todd D. Still und David G. Horrell (Hg.), After the First urban Christians: the Social-Scientific Study of Pauline Christianity Twenty-Five Years Later (New York 2009), 36-59; vgl. auch Bruce W. Longenecker, Remember the Poor: Paul, Poverty, and the Greco-Roman World (Grand Rapids (MI) 2010). 
auf versteckte Hinweise und Interpolationen stützen. Wer genau den Brief des Paulus tatsächlich gelesen und gehört hat, lässt sich nicht eruieren.

Die ersten Menschen, die sich in Korinth von Paulus haben taufen lassen, sind Stephanas und sein Haus (1 Kor 16,15). Entsprechend ist Stephanas der „Erstling von Achaia“. 1 Kor 1,14-16 entnehmen wir, dass Krispus - der Apg 18,8 zufolge der Synagogenvorsteher ist - sich Paulus anschliesst und ebenfalls zusammen mit seinem Haus taufen lässt wie auch Gaius. Weitere Taufen hat Paulus seines Wissens nicht vorgenommen (1 Kor 1,16). Es ist aber auch nicht Paulus' primäres Ziel, möglichst viele selbst zu taufen, sondern seine Aufgabe sieht er in der Bekanntmachung des Evangeliums (1 Kor 1,17). Dabei will er nicht die Taufe abwerten, sondern an der ihm richtig erscheinenden Reihenfolge festhalten: Zuerst wird das Evangelium verkündet, dann folgt die Taufe. Die Verkündigung des Evangeliums ist das, was die Gemeinschaft begründen soll, worauf diese aufbauen soll. Der grundlegende Inhalt des Evangeliums bzw. Verkündigens ${ }^{55}$ besteht in der Osterbotschaft ${ }^{56}$.

In der Zeit seines Aufenthalts in Korinth dürfte sich um das Ehepaar Priska und Aquila, das auch Paulus bei sich aufnimmt, eine sogenannte Hausgemeinde bilden. Aus dieser erwächst eine christusgläubige Gruppe in Korinth. Überhaupt ist das Haus (oikos) unbestrittenermassen der primäre Ort, von dem aus der Glaube an Christus praktiziert wird und von dem aus er sich verbreitet. Bestehende Häuser bieten einen relativ geschützten Rahmen für Versammlungen, innerhalb derer auch das Herrenmahl gefeiert wird. Nimmt man an, dass die Gruppe nach gängigem antikem Muster ein

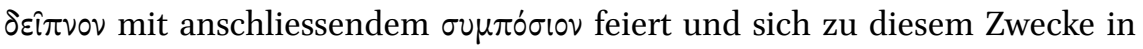
einem Triclinium versammelt, ergibt sich eine geschätzte Zahl von ca. zehn Kernmitgliedern. ${ }^{57}$ Diese Schätzung scheint allerdings zu gering, wenn man

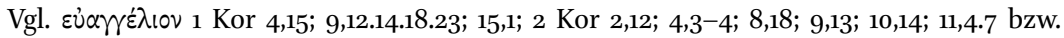

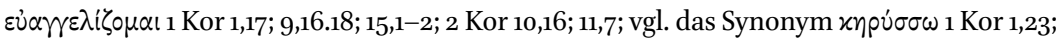
9,27; 15,11.12; 2 Kor 1,19; 4,5; 11,4 oder das Substantiv $\chi \eta ́ p u \gamma \mu \alpha ~ 1$ Kor 1,21; 2,4; 15,14.

$56 \quad 1$ Kor 2,1-5; 6,14; 15,1-4; 2 Kor 4,14; 5,15.

57 So die Argumentation im Abschnitt „The Size oft the Corinthian Group“ von Richard Last, The Pauline Church and the Corinthian Ekklēsia: Greco-Roman Associations in Comparative Context, MSSNTS 164 (New York 2016), 71-8o. Zum Vergleich von frühen Gruppen von Christusgläubigen mit Vereinen vgl. aus der Fülle jüngerer Literatur bspw. Richard S. Ascough, „What are they now Saying about Christ Groups and Associations?“, in: $C B R$ 13, Nr. 2 (2015), 207-244; Richard S. Ascough, „Voluntary Associations and the Formation of Pauline Christian Communities: Overcoming the objections“, in: A. Gutsfeld und Dietrich-Alex Koch (Hg.), Vereine, Synagogen und Gemeinden im kaiserzeitlichen Kleinasien (Tübingen 2006), 149-183; Eva Ebel, Die Attraktivität früher christlicher Gemeinden: Die Gemeinde von Korinth im Spiegel griechisch-römischer Vereine, WUNT 178 (Tübingen 2004); Harland, Dynamics of Identity in the World of the Early Christians; 
bedenkt, dass Paulus erwähnt, ausser Crispus, Gaius, Stephanas und dessen Haus keine Menschen getauft zu haben (1 Kor 1,14-16), und diese zugleich als wenige hinstellt gegenüber all den Menschen, die er eben nicht selbst getauft hat. Zu höheren Schätzungen kommt man über Mitgliedschaftslisten von Vereinen. ${ }^{58}$ Eine Obergrenze ergibt sich gleichwohl aus dem beschränkten Platz für Versammlungen. Für Korinth hat Murphy O'Connor festgestellt, dass die durchschnittliche Grösse der Atrien in den vier noch vorhandenen Häusern aus römischer Zeit in Korinth $55 \mathrm{~m}^{2}$ und die der Triklinien $36 \mathrm{~m}^{2}$ beträgt. Seiner Schätzung nach können sich hier maximal 50, aber eher nur $3^{0-}$ 40 Menschen versammeln. ${ }^{59}$ Eine höhere Zahl schätzen Caroline Osiek und D. L. Balch mit dem Argument, dass ausser in Triklinien auch im Peristyl oder in offenen Gärten gefeiert werden kann. ${ }^{60}$ Klauck rechnet gar mit einer Grösse von bis zu 200 Mitgliedern zur Zeit von Paulus' Aufenthalt. ${ }^{61}$ Mehr können es kaum sein, da sie sich noch in einem Raum zu treffen vermögen (1 Kor 14,23). Neben den Vollversammlungen wird es in Korinth auch verschiedene Hausgemeinschaften und damit unterschiedliche Versammlungstypen geben. ${ }^{62}$ Der in 1 Kor 14,23 erwähnte Raum dürfte sich im Hause des Gaius befinden, von dem in Röm 16,23 gesagt wird, dass er die ganze Versammlung gastlich

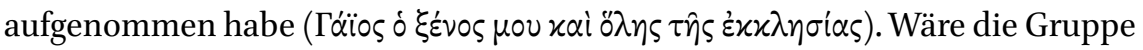
deutlich grösser, würde sie vermutlich auch im Bericht des aufmerksamen Beobachters Pausanias, der 100 Jahre nach Paulus in Korinth war, nicht fehlen.

Nach Paulus' Gründungsaufenthalt scheint der christusgläubige 'Iovð®îos Apollos in Korinth zu wirken (1 Kor 3,6). Apollos ist - wie Paulus selbst -

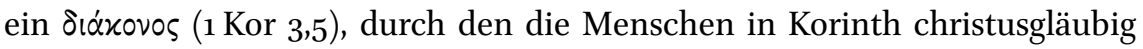
geworden sind. Paulus gibt an, dass er Apollos zu einem weiteren Aufenthalt in Korinth hat motivieren wollen, darin aber gescheitert ist (1 Kor 16,12). Auch nach dem Wirken des Apollos sieht sich Paulus explizit als Schirmherr der Gemeinschaft in Korinth. Dies geht beispielsweise daraus hervor, dass er die Menschen in Korinth als seine Kinder ( $\tau \dot{\varepsilon} \kappa \nu \alpha \mu \nu \nu \dot{\alpha} \gamma \alpha \pi \eta \tau \dot{\alpha}, 1$ Kor 4,14)

John S. Kloppenborg, „Graeco-Roman Thiasoi, the Corinthian Ekklesia at Corinth, and Conflict Management", in: Ron Cameron und Merrill P. Miller (Hg.), Redescribing Paul and the Corinthians, ECIL 5 (Atlanta 2011), 187-218; John S. Kloppenborg, „Membership Practices in Pauline Christ Groups", in: ECIL 4, Nr. 2 (2013), 183-215.

$5^{8}$ Vgl. ebd.

59 Vgl. Murphy-O'Connor, St. Paul's Corinth, 178-185. In diesem Rahmen bewegen sich auch die Schätzungen von Kloppenborg, „Membership Practices in Pauline Christ Groups“.

6o Vgl. Carolyn Osiek und David L. Balch, Families in the New Testament World: Households and House Churches, 3. Druck, The family, religion, and culture (Louisville (KY) 2000), 203.

$61 \quad$ Vgl. Klauck, 1. Korintherbrief, 8.

62 Vgl. Hans-Josef Klauck, Hausgemeinde und Hauskirche im frühen Christentum, SBS 103 (Stuttgart 1981), 35-41. 
bezeichnet und sich damit als Vater darstellt (vgl. 2 Kor 6,13; 12,14). ${ }^{63}$ Oder er betont, dass er für die Menschen in Korinth der Apostel ist und sie das Siegel seines Gesandtseins (1 Kor 9,2).

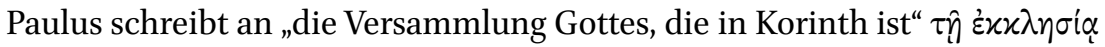

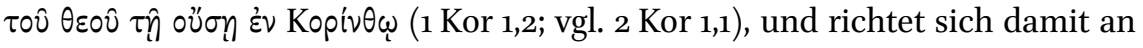
eine Gruppe, die er trotz vorhandener Streitigkeiten (1 Kor 1,11) noch als Einheit versteht (vgl. nur die Metaphorik vom einen Leib und von den vielen Gliedern in 1 Kor 12). ${ }^{64}$ Zudem spricht er die Adressaten als ein Kollektiv von $\alpha \dot{\delta} \varepsilon \lambda \varphi{ }^{\prime}$ an (1 Kor 1,10.11.26; 2,1; 3,1; 4,6; 7,24.29; 10,1; 11,33; 12,1; 14,6.20.26.39; 15,1.31.50.58; 16,15; vgl. 2 Kor 1,8; 8,1; 13,11). Offen bleibt, ob Paulus sein Schreiben tatsächlich an die gesamte Gruppe richtet oder ob er als implizite Leser eher ein Segment derselben im Blick hat.

Der Brief könnte auch von einem kleineren Kreis - wie beispielsweise einer informellen oder formellen, aber nicht als solchen bezeichneten Gemeindeleitung - oder gar von einer einzelnen Person zur Kenntnis genommen werden, die dafür zu sorgen hat, dass die Anweisungen von Paulus zur Umsetzung kommen.

Nur in 1 Thess 5,27 findet sich ein direkter Hinweis zum Umgang mit dem Brief, dass er nämlich „allen Brüdern“ vorgelesen werden solle. Dieser Hinweis am Briefende zeugt allerdings eher davon, dass nicht automatisch zu erwarten ist, dass der Brief in der Versammlung vorgetragen wird. Auch in Kol 4,16 und Off 22,18-19 findet sich jeweils ein Hinweis zum Umgang mit Briefen. Die Tatsache, dass der Hinweis sich jeweils am Briefende befindet, deutet darauf hin, dass vermutlich zuerst eine Einzelperson den Brief liest und dann entscheidet, wie sie damit weiter zu verfahren hat. Weiter ist zu fragen, wem der Brief sonst noch vorgelesen wird. Dass Paulus beim Schreiben des 1 Kor auch Frauen im Blick hat, lässt sich aufgrund einiger textimmanenter Hinweise annehmen. ${ }^{65}$ So spricht Paulus Frauen an, die verwitwet sind $(1$ Kor 7,8$)$ oder deren Männer nicht zu den Christusgläubigen zählen (1 Kor 7,13-16). Auch 1 Kor 11,3-16 lässt insbesondere mit den Bestimmungen über die Hierarchien zwischen Mann und Frau und mit denjenigen hinsichtlich einer Kopfbedeckung darauf schliessen, dass Frauen an Versammlungen teilnehmen. Auf den sozialen Hintergrund gibt 1 Kor 7,20-21

63 Zur Vatermetaphorik vgl. Gerber, Paulus und seine, Kinder', 351-426.

64 Die Überschrift zum Brief kommt erst später dazu und stammt von einem Abschreiber, der bereits eine Sammlung von Paulusbriefen gekannt haben muss.

65 Die folgenden Überlegungen stützen sich auf einen Vortrag von Julia Snyder, „An ,Ethics for Everyone?? Social Diversity and Paul's Target Audience" (gehalten im Construction of Christian Identities Seminar am Annual Meeting der Society of Biblical Literature in Boston am 19.11.2017). 
einen Hinweis mit der Anordnung, dass jeder in seinem Stand verbleiben soll. Dass Sklaven explizit genannt werden, lässt vermuten, dass solche zur Versammlung zählten.

Plausibel ist auch die Annahme, dass Paulus sich an diejenigen innerhalb der Gruppe wendet, die er für die in 1 Kor zahlreich genannten Missstände als verantwortlich ansieht bzw. die Anfragen an ihn gerichtet haben. Wenn dem so ist, bedeutet dies, dass der Brief eher als Dialog mit diesen bestimmten Gruppenangehörigen zu lesen und weniger als eine allgemeine Abhandlung anzusehen ist. Durch die Aufnahme des Briefes in den neutestamentlichen Kanon ist seine ursprüngliche Ausrichtung untergegangen, und er wird stattdessen als autoritativer Text verstanden, der sich an alle Getauften richtet.

\section{Zusammenfassung}

Für eine Beschreibung der impliziten Adressatenschaft finden sich in 1 Kor, aber auch in anderen Briefen zahlreiche textimmanente Hinweise. Die Zielkultur des paulinischen Vermittlungsgeschehens sind ganz pauschal „die Völker“, d. h. die gesamte nicht jüdische Menschheit. Dies ergibt sich aus Paulus' Auftrag, als Apostel für die Völker die Nicht-Iovס̊aio für sein Evangelium zu gewinnen. Die Ausrichtung auf eine primär pagane Adressatenschaft wird an verschiedenen Stellen deutlich. Einerseits spricht Paulus sie direkt als solche an, andererseits ist das Aushandeln mit den "drei Säulen“ von Bedeutung für die Ausrichtung seiner Mission. Es klärt die primäre Ausrichtung auf die Völker hin und damit auch die Rollen des Paulus und seiner Mitarbeitenden.

Zahlreiche textimmanente Hinweise lassen darauf schliessen, dass Paulus grundsätzlich eine synagogennahe Klientel im Blick hat. Um die Adressatinnen und Adressaten aus den Völkern zu erreichen, muss er zusammen mit seinen Mitarbeitenden die eigenen Überzeugungen auf eine Weise vermitteln, die für die Adressatinnen und Adressaten verständlich ist. Er lehnt sich häufig an jüdische Schriften an oder zitiert diese in seinen Argumentationen, was aufzeigt, dass er sich innerhalb der Denkstrukturen seines angestammten Orientierungssystems bewegt. Zugleich greift er - was noch zu zeigen sein wird - auf Bilder zurück, die im Alltag paganer Menschen eine zentrale Rolle spielen, und verleiht ihnen zugleich eine neue Bedeutung. Da Nicht-

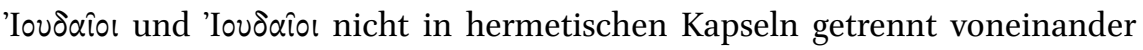
leben, sondern in unterschiedlichen Graden interagieren, dürfte es auch für

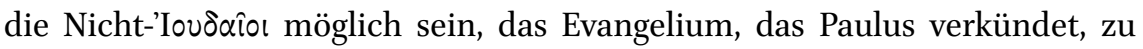
begreifen - insbesondere für Menschen, die bereits eine gewisse Nähe zum Judentum aufweisen. 
Nach der Ausrichtung des Vermittlungsgeschehens auf die pagane Menschheit insgesamt, wie sie in verschiedenen Paulusbriefen repräsentiert wird, hat sich der Blick spezifisch auf die Gruppe gerichtet. Die im Brief an die Gemeinde in Korinth angesprochene Adressatenschaft lebt in einer grossen, römisch geprägten, pulsierenden und hinsichtlich ihrer Bevölkerung sehr heterogenen Stadt. Diese ist geprägt von florierendem Handel, diversen Kulten, weltanschaulicher Offenheit und mit den Isthmischen Spielen ein bedeutender Ort kulturellen Lebens.

Die namentlich genannten Christusgläubigen deuten zusammen mit anderen Hinweisen - wie beispielsweise eine direkte Anrede auf die Herkunft aus den Völkern - auf eine primär pagane Adressatenschaft. Daneben zählen

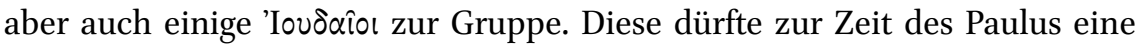
Grösse von einigen Dutzend bis max. 200 Mitglieder haben, und es ist innerhalb ihrer mit erheblichen sozialen Unterschieden zu rechnen. Wer von den Gruppenangehörigen die Paulusbriefe tatsächlich gelesen bzw. vorgelesen bekommen hat, bleibt offen. Dieser Aspekt des Transfergeschehens kann leider nicht abschliessend geklärt werden.

Die Beschreibung der paulinischen Adressatenschaft im Allgemeinen und der korinthischen Gruppe im Besonderen ist die Grundlage, auf der die Selbstdarstellung des Apostels als interkulturellen Vermittler in 1 Kor 9,19-23 und darauf folgend in 1 Kor 9,24-27 die implizite und zugleich konkrete Umsetzung dieses Anspruchs untersucht werden kann. 